\section{P133 HLA-ANTIGENS AND DISEASE MANIFESTATION IN A COHORT OF 600 SOUTHERN FRENCH PATIENTS WITH PSORIATIC ARTHRITIS}

\author{
${ }^{1} \mathrm{E}$ Massy, ${ }^{2} \mathrm{C}$ Picard, ${ }^{2} \mathrm{C}$ frassati, ${ }^{2} \mathrm{P}$ pedini, ${ }^{3} \mathrm{M}$ martin, ${ }^{3} \mathrm{l}$ Auger, ${ }^{1,3} \mathrm{~J}$ Roudier, \\ ${ }^{1,3} \mathrm{~N}$ Balandraud*. ${ }^{1}$ Rheumatology, Hôpital Sainte Marguerite; ${ }^{2}$ Etablissement Français du \\ sang, EFS; ${ }^{3}$ UMRs 1097 Immunogénétique Des Maladies Auto Immunes, INSERM and Aix \\ Marseille University, MARSEILLE, France
}

\subsection{6/annrheumdis-2018-EWRR2018.146}

Introduction Currently, there is no biomarker available to diagnose psoriatic arthritis (PsoA). Genome wide association studies showed that the majority of PsoA loci are shared with PsoC. ${ }^{1}$ However, PsoA has a strong familial predisposition, more so than PsoC as was shown in an Icelandic population. ${ }^{2}$ In the GWAS performed in UK and Germany, the strongest genetic locus is located within the major histocompatibility complex (MHC). ${ }^{3,4}$ No studies have been performed in southern Europe

Objectives The primary objective of our study was to focus on the HLA class I and II alleles found in a cohort of 600 French patients with PsoA clinically well characterised, compared to a control cohort. The secondary objective was to compare two clinical subsets of PsoA, one axial and one peripheral, to test which genotype determines these phenotypes.

Methods 600 patients from the Rheumatology department, St. Marguerite's Hospital, Marseilles, who fulfilled the CASPAR criteria for PsoA, underwent clinical, radiographic and laboratory investigations. HLA Class I and Class II alleles were genotyped. A cohort of 2346 healthy blood donors (HBD) was also tested.

Results

- Comparison between the PsoA population and controls showed one set of alleles significantly associated with PsoA; HLA-B*27, B*21(B*50), $C * 06$ and with a weak significance HLA-A*01, A*25, B*13.

- Within the PsoA population, two genetically different subsets determine two different clinical subtypes: Peripheral disease was significantly associated with $-C^{* 0} 06$ in disequilibrium linkage with $-B^{*} 13$ and $-D R * 07$, independently associated with HLA-A*01, $-B * 21$ and $-B * 17$. Axial disease was significantly associated with HLA-B*27 in disequilibrium linkage with $C^{*} 01$ and $-C^{*} 02$.

Conclusions This is the first and the largest study ever realised in southern Europe. It lead to conclude that PsoA is genetically heterogeneous. We found that PsoA is divided in two genetically and phenotypically relevant subgroups, one axial group with HLA-B*27 predominance very close to Ankylosis spondylitis and one peripheral group with HLA-C*06 predominance. Other HLA alleles within the MHC are also implied. The clinical utility of HLA typing in PsoA should be further addressed in larger studies.

\section{REFERENCES}

1. Stuart PE, Nair RP, Tsoi LC, et al. Genome-wide association analysis of psoriatic arthritis and cutaneous psoriasis reveals differences in their genetic architecture. Am J Hum Genet 2015;97:816-36. doi:10.1016/j.ajhg.2015.10.019

2. Chandran V, Schentag CT, Brockbank JE, et al. Familial aggregation of psoriatic arthritis. Ann Rheum Dis 2009:68:664-7. doi:10.1136/ard.2008.089367
3. Rahman P, Elder JT. Genetics of psoriasis and psoriatic arthritis: A report from the GRAPPA 2010 annual meeting. I Rheumatol 2012;39:431-3. doi:10.3899/ jrheum. 111242

4. Bowes J, Budu-Aggrey A, Huffmeier U, et al. Dense genotyping of immune-related susceptibility loci reveals new insights into the genetics of psoriatic arthritis. Nat Commun 2015:6:6046. doi:10.1038/ncomms7046

Disclosure of interest None declared

\section{P134 THE LYMPHATIC SYSTEM PLAYS AN IMPORTANT ROLE IN THE MIGRATION OF PATHOGENIC: T-CELLS TOWARDS SYNOVIAL JOINTS AND ENTHESES IN PSORIASIS}

${ }^{1} \mathrm{D}$ Verhaegh, ${ }^{2} \mathrm{E}$ Prens, ${ }^{1} \mathrm{~A}$ Mus, ${ }^{1} \mathrm{P}$ Asmawidjaja, ${ }^{1} \mathrm{~N}$ Davelaar, ${ }^{3} \mathrm{~A}$ Hofman, ${ }^{3} \mathrm{~J}-\mathrm{B}$ Jaquet, ${ }^{4} \mathrm{M}$ Kok, ${ }^{5} \mathrm{~S}$ Tas, ${ }^{6} \mathrm{H}$ Yagita, ${ }^{1} \mathrm{~J}$ Hazes, ${ }^{1} \mathrm{E}$ Lubberts, ${ }^{4} \mathrm{R}$ Bisoendial*. ${ }^{1}$ Rheumatology; ${ }^{2}$ Dermatology, Erasmus University Medical Centre; ${ }^{3}$ Plastic Surgery; ${ }^{4}$ Rheumatology, Maasstad Hospital, Rotterdam; ${ }^{5}$ Rheumatology, Academic Medical Centre, Amsterdam, Netherlands; ${ }^{6}$ Immunology, Juntendo University School of Medicine, Tokyo, Japan

\subsection{6/annrheumdis-2018-EWRR2018.147}

Introduction Psoriasis (PsO) is a common inflammatory skin disease that is characterised by acanthosis, oedema formation, immune cell infiltration, abnormal vascular proliferation, and remodelling of the lymphatic system. Up to $\sim 30 \%$ of $\mathrm{PsO}$ patients are affected by psoriatic arthritis, a devastating form of arthritis, which leads to disability and deterioration of quality of life. Thusfar, the transition from PsO to PsA, particularly the factors that determine the propensity of pathogenic T-cells in psoriasis to colonise synovial membrane in joints and entheses are poorly understood. Lymphatic endothelial cells (LECs), core components of the lymphatic vascular system and lymph nodes, may control the transmission of pathogenic $\mathrm{T}$-cells in $\mathrm{PsO}$ to other sites including synovial joints and entheseal regions by programming these T-cells in terms of their homing properties.

Objectives The focus of our proposal is to investigate whether LECs are capable of modulating the differentiation and homing receptor profile on CD4 memory T-cells.

Methods Human dermal lymphatic endothelial cells (LEC; $\left.0.5 \times 10^{4}\right)$, and fibroblast-like synoviocytes of a patient with PsA (FLS; $1.0 \times 10^{4}$ ) were pre-incubated for 3 days with PsA synovial fluid (PsA-SF; 0/10/20\% v/v). Then, LEC or FLS were co-cultured with $2.5 \times 10^{4}$ allogeneic $\mathrm{CD} 4{ }^{+} \mathrm{CD} 45 \mathrm{RO}^{+} \mathrm{CD} 25^{-} \mathrm{T}$-cells that were sorted from peripheral blood mononuclear cells (PBMC) of 3 healthy donors with or without stimulation with $\alpha \mathrm{CD} 3 \quad(0.3 \mu \mathrm{g} / \mathrm{ml})$ and $\alpha \mathrm{CD} 28(0.4 \mu \mathrm{g} / \mathrm{ml})$.

Results By means of flow-cytometry based phenotypical analysis, dermal LECs appear to suppress the generation of Th17 cells from stimulated CD4 memory T-cells of healthy donors, as compared to co-culture experiments involving synovial fibroblasts. Initial studies to the underlying mechanisms suggest that blocking the non-canonical NF- $\mathrm{B}$ pathway downstream of the lymphotoxin beta receptor (LT $\beta R$ ) impairs the capacity of the LECs to suppress Th17 generation. In addition, Deltalike ligand 4 (DLL4) is differentially expressed in LECs and synovial fibroblasts after 3 days of co-culture. Earlier studies have shown that DLL4 may induce RORyt expression. Blocking DLL4 using a monoclonal antibody also impairs the capacity of the LECs to suppress Th17 generation.

Conclusions In our co-culture setup, LECs suppress Th17 generation from stimulated CD4 memory T-cells, and are thus directly involved in T-cell differentiation. The LT $\beta R /$ non- 


\section{Abstracts}

canonical NF- $\kappa$ B pathway and downstream DLL4-Notch1/4 may be involved in LEC mediated modulation of T-cell homing and deserves further exploration.

Disclosure of interest None declared 